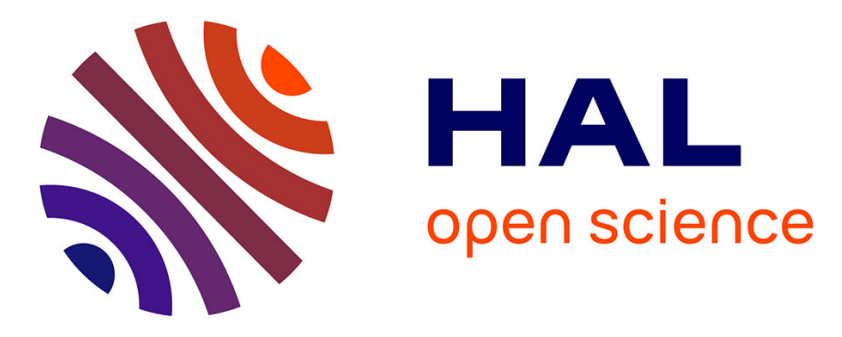

\title{
Molecular line lists for modelling the opacity of cool stars
}

Jonathan Tennyson, Gregory Harris, Bob Barber, Santina La Delfa, Boris A Voronin, Yakov V Pavlenko

\section{- To cite this version:}

Jonathan Tennyson, Gregory Harris, Bob Barber, Santina La Delfa, Boris A Voronin, et al.. Molecular line lists for modelling the opacity of cool stars. Molecular Physics, 2007, 105 (05-07), pp.701-714. 10.1080/00268970701196983 . hal-00513080

\section{HAL Id: hal-00513080 \\ https://hal.science/hal-00513080}

Submitted on 1 Sep 2010

HAL is a multi-disciplinary open access archive for the deposit and dissemination of scientific research documents, whether they are published or not. The documents may come from teaching and research institutions in France or abroad, or from public or private research centers.
L'archive ouverte pluridisciplinaire HAL, est destinée au dépôt et à la diffusion de documents scientifiques de niveau recherche, publiés ou non, émanant des établissements d'enseignement et de recherche français ou étrangers, des laboratoires publics ou privés. 


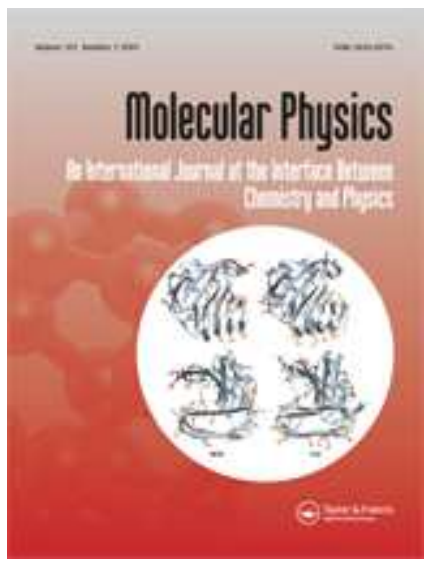

Molecular line lists for modelling the opacity of cool stars

\begin{tabular}{|r|l|}
\hline Journal: & Molecular Physics \\
\hline Manuscript ID: & TMPH-2006-0094.R1 \\
\hline Manuscript Type: & Full Paper \\
\hline Dathor: & 23-Dec-2006 \\
\hline Complete List of Authors: & $\begin{array}{l}\text { Tennyson, Jonathan; University College London, Chemistry } \\
\text { Harris, Gregory; UCL } \\
\text { Barber, Bob; UCL } \\
\text { la Delfa, Santina; UCL } \\
\text { Voronin, Boris; UCL } \\
\text { Pavlenko, Yakov; National Academy of Sciences }\end{array}$ \\
\hline Keywords: & spectroscopy, rotation-vibration, computation \\
\hline \multicolumn{2}{|c|}{} \\
\hline \hline $\begin{array}{l}\text { Note: The following files were submitted by the author for peer review, but cannot be converted } \\
\text { to PDF. You must view these files (e.g. movies) online. }\end{array}$ \\
\hline \begin{tabular}{l} 
final2.tex \\
\hline
\end{tabular}
\end{tabular}

\section{scholaroNE" \\ Manuscript Central}




\title{
Molecular line lists for modelling the opacity of cool stars
}

\author{
JONATHAN TENNYSON $\dagger^{*}$, GREGORY J. HARRIS $\dagger$, ROBERT J. BARBER $\dagger$, \\ SANTINA LA DELFA $\dagger$, BORIS A. VORONIN $\dagger, \$$, B. M. KAMINSKY $\ddagger$ and Ya. V. \\ PAVLENKO $\ddagger$ \\ $\dagger$ Department of Physics and Astronomy, University College London, \\ Gower St., London WC1E 6BT, U.K. \\ $\ddagger$ Main Astronomical Observatory, National Academy of Sciences, \\ Zabolotnoho 27, Kyiv-127 03680, Ukraine. \\ $\$$ Institute of Atmospheric Optics, SB, Russian Academy of Sciences, Tomsk, Russia \\ (Received 00 Month 200x; In final form 00 Month 200x)
}

\begin{abstract}
The behaviour of cool stars is largely governed by molecular absorptions in their outer atmospheres. At the temperatures involved $(1000-4000 \mathrm{~K})$, the number of transitions involved can be vast. The underlying physics of the problem is outlined and the status attempts to address the demands of stellar models discussed. Progress on calculations of line lists for stellar models at UCL is described. Specific examples of involving water, $\mathrm{HCN} / \mathrm{HNC}, \mathrm{C}_{3}, \mathrm{H}_{3}^{+}$and $\mathrm{HDO}$ are given. Outstanding needs are summarised.
\end{abstract}

\section{Introduction}

Molecular processes have a significant influence on many aspects of our Universe [1]. It is well established that the huge "molecular" clouds out of which stars form have a detailed and unique chemistry [2]. What is often not so well appreciated is that for the majority of stars it is also molecular processes that govern how they behave and evolve. Until recently these cooler stars have received little attention as they are much less readily studied than their significantly brighter, hot, atomic counterparts.

The light that reaches us from a star is moderated by the star's atmosphere through which radiation must travel in order to escape. A star's spectrum, and many other properties of the star, are determined by the ability of the species in its atmosphere to absorb light. This ability to absorb is known as the opacity and only through using a proper opacity function is it possible to obtain reliable stellar models. For hot stars, where the processes in ques-

* Corresponding author, e-mail: j.tennyson@ucl.ac.uk 
tion involve atoms and atomic ions, detailed $a b$ initio quantum mechanical calculations [3-5] have been used to populate data compilations [6]. These calculations have essentially solved the opacity problem for hot stars.

For hot stars the photon-induced transitions are usually classified as three types: bound-bound (electronic excitation), bound-free (photoionisation) and free-free (inverse Bremsstrahlung). The processes determining the opacities of cool stars are considerably more varied [7]. In this paper we report progress in trying to meet the demands placed on laboratory astrophysicists attempting to provide the spectroscopic data necessary to construct molecular opacities.

\section{Underlying physics}

\subsection{Cool stars}

The sun, with a surface temperature of $5785 \mathrm{~K}$, lies at the boundary between hot (entirely atomic) and cold (molecule containing) stars. Its atmosphere contains significant quantities of diatomic species such hydrogen, carbon monoxide and $\mathrm{CH}$. Sunspots, which are much cooler, contain other molecules including water [8].

In contrast to the interstellar medium, the chemistry of stellar atmospheres essentially follows the laws of thermodynamic equilibrium, as expressed by the Saha equation, for the given molecular composition and temperature. Of course the dominant species in such atmospheres are hydrogen and helium, however the most important property for determining the chemical composition of the star is the ratio of carbon to oxygen. This is because the strongly bound CO molecule rapidly forms in the stellar environment and whether there is a surplus of oxygen or carbon that remains has a profound effect on the properties of the star $[1,9]$.

Cool stars which are richer in $\mathrm{O}$ than $\mathrm{C}$ are know as $\mathrm{M}$ dwarfs. These are by far the most common stars in the galaxy. $M$ dwarfs have atmospheric temperatures in the 2000 to $3500 \mathrm{~K}$ range; while hotter $\mathrm{K}$ dwarfs, spanning 3500 to $5000 \mathrm{~K}$, show weaker molecular features. There remain significant uncertainties in the precise temperatures. The very strong molecular absorption features, largely due to water and $\mathrm{TiO}$ molecules, in the spectra of $\mathrm{M}$ dwarf means that the spectra bear little resemblance to a Planck function. Thus the temperature can only be found by comparison with detailed models and, thus far, no model has been entirely successful in reproducing these spectra. This problem is often blamed on the quality of the spectroscopic data used in the models (eg $[10,11])$ but the need to make assumptions about the abundances of the various atomic species also adds a significant indeterminacy.

Cool stars with more carbon than oxygen are known as carbon stars or C dwarfs. As can be guessed from terrestrial experience, the chemistry of carbon 
stars is more complicated than that of $M$ dwarfs and a significant number of different carbon containing species are thought to contribute to their opacity. Easier to see, and therefore better studied, than C dwarfs are C-giants, or red giants, which are old, cool stars which have completed the hydrogen-burning phase of their evolution. C giants can have luminosities 1000 times greater than the Sun. As discussed below there are no complete line lists available for some of the key species found in C-star atmospheres.

There is also a class of dwarf stars, known as S dwarfs, for which the $\mathrm{C}$ and $\mathrm{O}$ abundances are approximately equal. These stars are extremely rare and will not be considered further. However there is one further class of dwarf stars which do not follow the above chemistries.

Most stars in the galaxy contain quantities of elements heavier than helium (called metals by astronomers) which are close to those found in the Sun. However, big bang nucleosynthesis models predict that the Universe first formed with hydrogen, helium and trace quantities of lithium and beryllium. The heavy elements were produced later by fusion and neutron capture in stars and supernovae. Astronomical surveys are discovering increasing numbers of old and very metal deficient stars (eg [12]). As metals are more readily ionised than hydrogen and helium the low abundances of metals result in low abundances of free electrons. These metal-poor stars were formed out of primordial matter before significant quantities of elements heavier than helium had been synthesised. Such stars have a unique chemistry based on hydrogen and helium [13].

Cooler and less massive than the dwarf stars discussed above are brown dwarfs whose interiors do not get hot enough for sustained hydrogen burning. The spectra of these failed stars show similarities with the spectra of gas giant planets. The status of atomic and molecular opacities for brown dwarfs has recently been reviewed by Sharp and Burrows [14].

A final class of objects in which molecular processes play an important role are ultra-cool white dwarfs. White dwarfs are the remains of dead intermediate and low mass stars. The have very high surface gravities, and initially form with very high surface temperatures $(\sim 100000 \mathrm{~K})$. However, over billions of years they gradually radiate their thermal energy away and cool down. The coolest known white dwarfs have surface temperatures in the region of $2500-3500 \mathrm{~K}$. Due to the extremely high surface gravity, heavy elements gravitationally settle, so that the surface of white dwarfs is almost entirely hydrogen (DA) or almost entirely helium (non-DA). The ionisation equilibrium in ultra-cool hydrogen rich white dwarfs is dominated by $\mathrm{H}_{3}^{+}$[15], and by $\mathrm{HeH}^{+}$ in helium rich white dwarfs [16]. 


\section{$2.2 \quad$ Molecular processes}

The most apparent way in which molecules affect the atmospheres of cool stars is by the direct absorption of radiation, with particular emphasis on the whole infrared and red end of the visible as it is in these regions that the black body curves of cool stars have significant flux. When attempting to address the laboratory data requirements of models of these spectra it is useful to categorise the various processes according to their underlying physics.

Given that $\mathrm{H}_{2}$ and $\mathrm{CO}$ are the most abundant molecular species in the atmospheres of cool stars, one important class of molecules is closed shell diatomics. For these species their contribution to the line opacity is given by rotation-vibration transitions. It should be noted that since the $\mathrm{H}_{2}$ transitions are only weak quadrupole ones, collision induced absorption is also very important for this species [17]. The opacity of such species is in general fairly well understood and CO spectra, for example, are now used routinely to obtain information about cool stars (eg [18]). Recently the potential importance of other less well studied diatomic species has been recognised leading to the calculation of opacities for metal halides [19] and the molecular ion $\mathrm{HeH}^{+}[20]$.

Much more complicated than these closed shell diatomics are the open shell diatomic species formed by transition metals. These species are important because they have electronic bands which lie at long wavelengths (near infrared or visible) where they can absorb light from the star. For M dwarfs the TiO molecule has plays a particularly important role [21]. The spectrum of TiO is very complicated with many perturbations and interactions [22], however its opacity seems to be now reasonably well characterised using a mixture of $a b$ initio calculations and laboratory experiments [23,24], see fig. 7 of Pavlenko et al. [25].

For other similar open shell species there remains significant work to be done. For example the highly complicated laboratory spectrum of the $\mathrm{FeH}$ radical has been intensively studied by Brown and co-workers over many years [26-39] and by others [40]. The particular case of $\mathrm{CrH}$ and $\mathrm{MgH}$ will be discussed further below.

For molecules larger than diatomic it is the rotation-vibration spectrum of closed shell species which have mainly to be considered. For M dwarfs water is of particular importance [41]. For carbon stars one needs to consider triatomic species such as HCN and $\mathrm{HNC}$ [42], and $\mathrm{C}_{3}$ [10]. For metal free stars $\mathrm{H}_{3}^{+}$is potentially important [13]. As described below, comprehensive line lists for such systems can be now be calculated with reasonable reliability using variational nuclear motion procedures.

For some dwarfs molecules larger than triatomics are known to form: acetylene and methane are thought to be particularly important for carbon stars [10] and ammonia for brown dwarfs, particularly the yet to be positively identified 
coolest brown dwarf stars which have been labelled Y dwarfs [43]. Given that the computed rotation-vibration line lists for triatomic species have contained between 10 and 500 million distinct transitions, line list for these polyatomic molecules will need to consider many billions of transitions. So far no comprehensive line list exists for any species larger than triatomic.

Molecules influence the structure of stars by means other than their spectra. In particular in cool metal poor stars the electrons provided by molecular ions can completely alter the structure of the star due to their indirect effect on the opacity which in this case is dominated by the continuum absorption of the $\mathrm{H}^{-}$anion. Under these circumstances, the use of correctly computed partition functions for $\mathrm{H}_{3}^{+}$[44] and $\mathrm{HeH}^{+}$[20], are important, see [15] and [16] respectively.

Finally it should be mentioned that the spectra of isotopically substituted molecules (isotopologues) can also be important for studies of the atmospheres of cool stars. Isotopic ratios can provide information about the evolutionary status of the stars, on the nature of nuclear processes in the stellar cores and on the physical state of the convective envelopes.

\section{Line list calculations}

The group at UCL has undertaken the systematic calculation of molecular line lists for modelling stellar opacities. In practise these line lists are often used for other applications ranging from modelling laboratory experiments $[45,46]$ to assigning laboratory spectra [47]. Below we present some recent results obtained from these line list calculations, some of which are still in progress.

\subsection{HCN and HNC.}

An extensive $a b$ initio linelist for the HCN and HNC system has been computed [48]. This linelist contains nearly 400 million transitions between 168000 energy levels, it extends up to $J=60$ in angular momentum and up to 18000 $\mathrm{cm}^{-1}$ above the zero point energy. The fundamental vibrational modes reproduce laboratory data to within $4 \mathrm{~cm}^{-1}$, except for then HNC stretching mode which deviates from experiment by $14 \mathrm{~cm}^{-1}$. The $a b$ initio line intensities reproduce laboratory data, usually, to within experimental error [49].

This linelist has been used to model the atmospheres and spectra of cool carbon rich giant stars [48]. The observed HCN absorption bands between 2.8 and $4.0 \mu \mathrm{m}$ in the stellar spectra are well reproduced. Furthermore the $\mathrm{HCN} / \mathrm{HNC}$ linelist has allowed the first identification of an HNC absorption feature in a stellar spectrum. This is the H-NC stretching mode at $2.8 \mu \mathrm{m}$ $\left(3600 \mathrm{~cm}^{-1}\right)$. However, at medium resolutions the structure and wavelength 


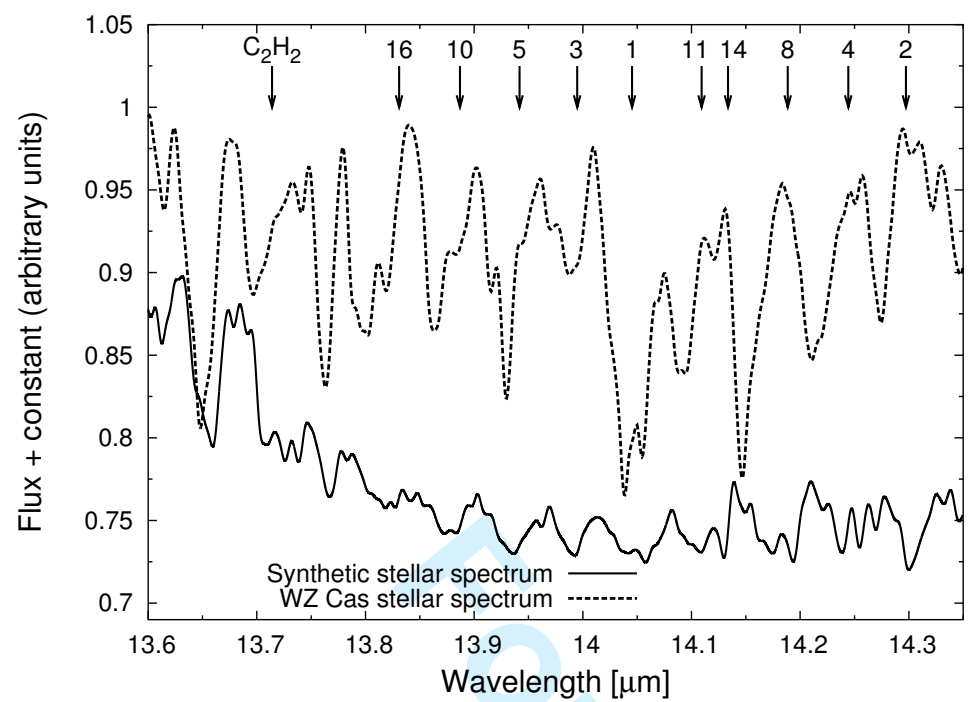

Figure 1. Observed and synthetic spectra of the carbon star WZ Cas. The synthetic spectra has been convolved with a Gaussian of half width at half maximum of $3.5 \times 10^{-3} \mu \mathrm{m}$. The position of the HCN Q-branches is indicated by an arrow and digit, see table 1 for wavelengths and quantum numbers. The $\mathrm{C}_{2} \mathrm{H}_{2}$ cis bending fundamental is also indicated. The synthetic spectra have been displaced from the observed spectra by 0.1 in arbitrary flux units.

of the Q branches of the parallel bands are poorly reproduced. This is a direct result of the inaccuracies of the energy levels in the $a b$ initio linelist.

To improve the reproduction of the observed HCN and HNC bands, experimental line frequency data has been used to determine energy levels. These laboratory determined energy levels have been incorporated into the $a b$ initio linelist replacing the $a b$ initio energy levels [50]. This improved linelist has also been used to model the atmospheres and spectra of carbon giant stars. The reproduction of the observed structure and frequency of the observed parallel bands at 3.55 and $3.85 \mu \mathrm{m}\left(2800\right.$ and $\left.2600 \mathrm{~cm}^{-1}\right)$ has been greatly improved [50].

Figures 1 and 2 show the observed spectra of the carbon star WZ Cas in the mid infrared, observed by Aoki et al. [51] using the Infrared Space Observatories (ISO) Short Wavelength Spectrometer (SWS). Also plotted are synthetic stellar spectra computed at $2800 \mathrm{~K}$, a surface gravity of $\log g=0$ in cgs units, and a $\mathrm{C}$ to $\mathrm{O}$ ratio of 1.006 . The synthetic spectra were convolved using a Gaussian profile to match the resolution of the ISO SWS spectrometer, which has a resolving power of roughly 2000 .

The 13.6 to $14.35 \mu \mathrm{m}$ region shown in figure 1 covers the centre of the HCN bending fundamental. Figure 2 covers the spectral range 20 to $21.9 \mu \mathrm{m}$, which corresponds to the region in which the Q branches of the HNC bending fundamental and its hot bands lie. In both figure 1 and 2 the position of 


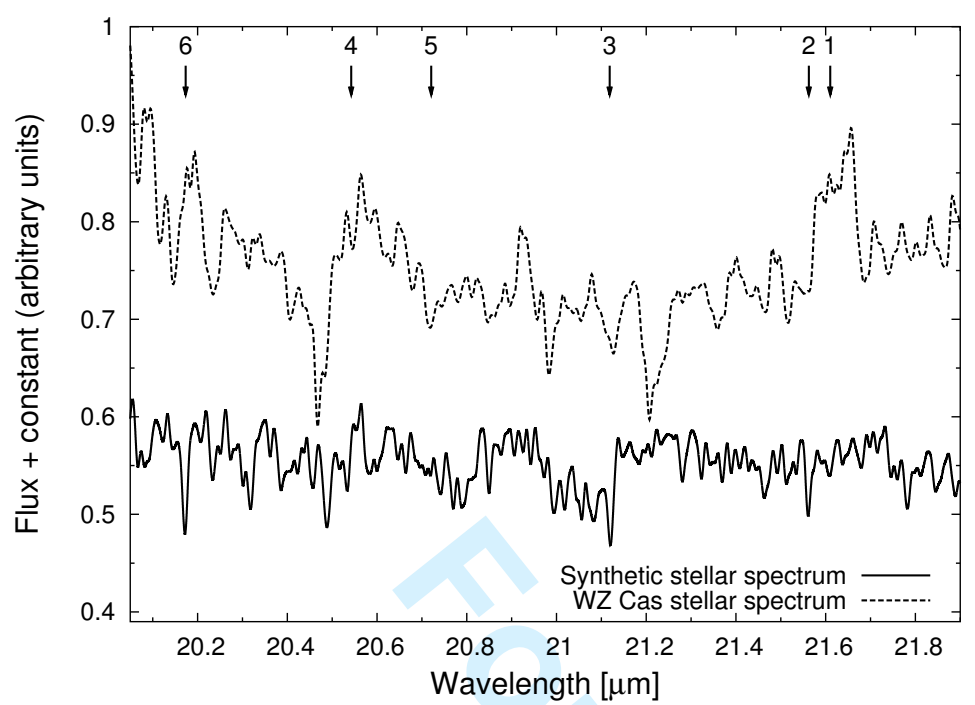

Figure 2. Observed and synthetic spectra of the carbon star WZ Cas. The synthetic spectrum has been convolved with a Gaussian of half width at half maximum of $5 \times 10^{-3} \mu \mathrm{m}$. The position of each of the HNC Q-branches is indicated by an arrow, frequencies and quantum numbers are listed in table 2 . The synthetic spectra have been displaced from the observed spectra by 0.4 in arbitrary flux units.

the lowest $J$ lines for the Q branches of the respective bands are indicated by arrows and an index number. The corresponding line frequencies and quantum numbers for each index number are listed in table 1 for figure 1 and table 2 for figure 2. The line frequencies in tables 1 and 2 are either based upon experimental data or are the results of $a b$ initio calculations, see Harris et al. [50] and references within. The aproxomate vibrational quantam numbers for $\mathrm{HCN}$ and $\mathrm{HNC}$ are $\mathrm{H}-\mathrm{CN}$ or $\mathrm{H}-\mathrm{NC}$ stretch $\left(v_{1}\right)$, doubly degenerate bend $\left(v_{2}\right)$, vibrational angular momentum $(l)$ and $\mathrm{C}-\mathrm{N}$ stretch $\left(v_{3}\right)$.

In figure 1 the most of the $\mathrm{Q}$ branches of the bending fundamental and its hot bands are evident in both the synthetic and the observed spectrum. For most of the absorption features in the observed spectrum there is a corresponding feature in the synthetic spectrum. However, the HCN absorption in the synthetic spectrum is saturated, the difference between the absorption at the centre and the edge of the $\mathrm{Q}$ branches is small. This is due to a very high abundance of HCN in the model atmosphere, so that all the flux emerging from the atmosphere in this spectral region comes from the atmospheric layers above the layers which contain HCN. Thus the changes in the HCN opacity have little affect upon the emergent stellar spectrum. Conversely the stellar spectrum does not appear to be saturated, the difference between the absorption at the centre and the edge of the Q branches is larger than for the observed spectrum.

Aoki et al. [52] also had problems reproducing the observed absorption in this 
spectral region, they suggested that this might be due to circumstellar HCN emission. Circumstellar gas which surrounds a asymptotic giant branch (AGB) stars, is invariably cooler than the stellar atmosphere. The circumstellar gas absorbs stellar radiation and re-emits it at longer wavelengths. So circumstellar gas is usually observed in emission at wavelengths longword of the mid infrared. As the circumstellar gas is cooler than the atmosphere, any HCN emission would come from the fundamental and the low lying hot bands. Thus the fundamental would appear in emission whilst the higher hot bands would appear in absorption. This is not observed, so for our models circumstellar emission will not provide a solution.

This problem may also be due to an incorrect stellar effective temperature, carbon to oxygen ratio or surface gravity. However, the temperature and $\mathrm{C} / \mathrm{O}$ ratio which we have used to compute these spectra, provide good fits to the longer wavelength regions. Missing molecular opacity could also be responsible for the poor agreement between the observed and synthetic spectra. The addition of a further molecular opacity sources often significantly alter both the structure of the model atmosphere and the synthetic spectra. $\mathrm{C}_{2} \mathrm{H}_{2}$ is known to be abundant in cool carbon stars, the wavelength of the cis bending fundamental is close to that of HCN at $13.71 \mu \mathrm{m}\left(729.2 \mathrm{~cm}^{-1}\right)$. The position of this fundamental is marked in figure 1 , and coincide with an absorption feature in the observed spectrum. However, no extensive data set for acetylene is currently in the public domain, so it has not been used in the computation of synthetic spectra. The inclusion of such data would be likely to significantly change the model atmosphere and synthetic spectrum.

It is difficult to make any definite identification of Q-branches of the HNC $\Delta v_{2}=1$ bands shown in figure 2 . There appears to be a large amount of absorption in this region which cannot be attributed to HNC, particularly at 20.45 and $21.25 \mu \mathrm{m}$ (489.0 and $470.6 \mathrm{~cm}^{-1}$ ), which is not reproduced in the synthetic spectra. There is weak HNC Q-branch absorption in the synthetic spectrum for the hot bands $2,3,4,5,6$, but little absorption which can be attributed to the fundamental. The reason for this is that the number of lines per unit wavelength for the fundamental is lower than for the hot bands. The HNC fundamental corresponds with an increase in flux in the observed spectrum. The increase may be due to emission from cool circumstellar HNC or more likely due to a gap between absorption features of unidentified species. The hot band with upper state $\left(0,2^{2}, 0\right)$ (labelled 3 in figure 2 ) appears shows the strongest absorption of all the bands in the synthetic spectrum. The is also a corresponding absorption feature in the observed spectrum, however it is not possible to confirm this absorption as HNC without positive identification of the other HNC Q-branches. Laboratory data for the excited HNC bending modes is far less extensive than for the HCN modes. For states with more than 2 quanta of bend no experimental data exists. Further work needs to be 
Table 1. Lines from the Q branches of the HCN $\Delta v_{2}=1$ fundamental and hot bands, with the lowest possible $J$. Here index refers to the index number marked on figure $1, P^{\prime \prime}$ is parity of the lower state and all line frequencies were determined using laboratory data.

\begin{tabular}{cccccccccccccc}
\hline index & $\lambda(\mu \mathrm{m})$ & $\nu\left(\mathrm{cm}^{-1}\right)$ & $J$ & $P^{\prime \prime}$ & $v_{1}^{\prime \prime}$ & $v_{2}^{\prime \prime}$ & $l^{\prime \prime}$ & $v_{3}^{\prime \prime}$ & $v_{1}^{\prime}$ & $v_{2}^{\prime}$ & $l^{\prime}$ & $v_{3}^{\prime}$ \\
\hline 16 & 13.8310 & 723.015 & 5 & $\mathrm{e}$ & 0 & 4 & 4 & 0 & 0 & 5 & 5 & 0 \\
- & 13.8316 & 722.982 & 5 & $\mathrm{f}$ & 0 & 4 & 4 & 0 & 0 & 5 & 5 & 0 \\
10 & 13.8869 & 720.105 & 4 & $\mathrm{e}$ & 0 & 3 & 3 & 0 & 0 & 4 & 4 & 0 \\
- & 13.8875 & 720.071 & 4 & $\mathrm{f}$ & 0 & 3 & 3 & 0 & 0 & 4 & 4 & 0 \\
5 & 13.9418 & 717.265 & 3 & $\mathrm{e}$ & 0 & 2 & 2 & 0 & 0 & 3 & 3 & 0 \\
- & 13.9418 & 717.265 & 3 & $\mathrm{f}$ & 0 & 2 & 2 & 0 & 0 & 3 & 3 & 0 \\
3 & 13.9940 & 714.592 & 2 & $\mathrm{e}$ & 0 & 1 & 1 & 0 & 0 & 2 & 2 & 0 \\
- & 13.9949 & 714.547 & 2 & $\mathrm{f}$ & 0 & 1 & 1 & 0 & 0 & 2 & 2 & 0 \\
1 & 14.0451 & 711.994 & 1 & $\mathrm{e}$ & 0 & 0 & 0 & 0 & 0 & 1 & 1 & 0 \\
11 & 14.1093 & 708.751 & 1 & $\mathrm{e}$ & 0 & 0 & 0 & 1 & 0 & 1 & 1 & 1 \\
14 & 14.1336 & 707.532 & 3 & $\mathrm{e}$ & 0 & 4 & 2 & 0 & 0 & 5 & 3 & 0 \\
- & 14.1336 & 707.532 & 3 & $\mathrm{f}$ & 0 & 4 & 2 & 0 & 0 & 5 & 3 & 0 \\
8 & 14.1886 & 704.794 & 2 & $\mathrm{e}$ & 0 & 3 & 1 & 0 & 0 & 4 & 2 & 0 \\
- & 14.1904 & 704.700 & 2 & $\mathrm{f}$ & 0 & 3 & 1 & 0 & 0 & 4 & 2 & 0 \\
4 & 14.2438 & 702.060 & 1 & $\mathrm{e}$ & 0 & 2 & 0 & 0 & 0 & 3 & 1 & 0 \\
2 & 14.2973 & 699.435 & 1 & $\mathrm{f}$ & 0 & 1 & 1 & 0 & 0 & 2 & 0 & 0 \\
12 & 14.4469 & 692.189 & 1 & $\mathrm{e}$ & 0 & 4 & 0 & 0 & 0 & 5 & 1 & 0 \\
7 & 14.5032 & 689.501 & 1 & $\mathrm{f}$ & 0 & 3 & 1 & 0 & 0 & 4 & 0 & 0 \\
6 & 14.5562 & 686.994 & 2 & $\mathrm{e}$ & 0 & 2 & 2 & 0 & 0 & 3 & 1 & 0 \\
- & 14.5581 & 686.902 & 2 & $\mathrm{f}$ & 0 & 2 & 2 & 0 & 0 & 3 & 1 & 0 \\
13 & 14.7702 & 677.039 & 2 & $\mathrm{e}$ & 0 & 4 & 2 & 0 & 0 & 5 & 1 & 0 \\
- & 14.7738 & 676.872 & 2 & $\mathrm{f}$ & 0 & 4 & 2 & 0 & 0 & 5 & 1 & 0 \\
9 & 14.8263 & 674.476 & 3 & $\mathrm{e}$ & 0 & 3 & 3 & 0 & 0 & 4 & 2 & 0 \\
- & 14.8263 & 674.477 & 3 & $\mathrm{f}$ & 0 & 3 & 3 & 0 & 0 & 4 & 2 & 0 \\
15 & 15.1056 & 662.005 & 4 & $\mathrm{e}$ & 0 & 4 & 4 & 0 & 0 & 5 & 3 & 0 \\
- & 15.1062 & 661.980 & 4 & $\mathrm{f}$ & 0 & 4 & 4 & 0 & 0 & 5 & 3 & 0 \\
\hline & & & & & & & & & & & \\
\hline
\end{tabular}

conducted in identifying the additional absorption, and any emission across this region.

\section{$3.2 \mathrm{H}_{3}^{+}$}

The molecular ion $\mathrm{H}_{3}^{+}$is destroyed both by dissociative recombination with electrons and by proton donation to most molecular species. This means that the formation of $\mathrm{H}_{3}^{+}$is only favoured in stars with low metal content. It has 
Table 2. Lines from the Q branches of the HNC $\Delta v_{2}=1$ fundamental and hot bands, with the lowest possible $J$. Here index refers to the index number marked on figure $2, P^{\prime \prime}$ is parity of the lower state, source refers to either a line frequency determined from laboratory data (lab) or an $a b$ initio calculation (ai).

\begin{tabular}{ccccccccccccccc}
\hline index & $\lambda(\mu \mathrm{m})$ & $\nu\left(\mathrm{cm}^{-1}\right)$ & $J$ & $P^{\prime \prime}$ & $v_{1}^{\prime \prime}$ & $v_{2}^{\prime \prime}$ & $l^{\prime \prime}$ & $v_{3}^{\prime \prime}$ & $v_{1}^{\prime}$ & $v_{2}^{\prime}$ & $l^{\prime}$ & $v_{3}^{\prime}$ & source \\
\hline- & 20.1733 & 495.705 & 3 & $\mathrm{f}$ & 0 & 2 & 2 & 0 & 0 & 3 & 3 & 0 & $\mathrm{ai}$ \\
6 & 20.1734 & 495.702 & 3 & $\mathrm{e}$ & 0 & 2 & 2 & 0 & 0 & 3 & 3 & 0 & $\mathrm{ai}$ \\
4 & 20.5424 & 486.798 & 1 & $\mathrm{e}$ & 0 & 2 & 0 & 0 & 0 & 3 & 1 & 0 & $\mathrm{ai}$ \\
5 & 20.7209 & 482.604 & 2 & $\mathrm{e}$ & 0 & 2 & 2 & 0 & 0 & 3 & 1 & 0 & $\mathrm{ai}$ \\
- & 20.7265 & 482.475 & 2 & $\mathrm{f}$ & 0 & 2 & 2 & 0 & 0 & 3 & 1 & 0 & $\mathrm{ai}$ \\
3 & 21.1186 & 473.517 & 2 & $\mathrm{e}$ & 0 & 1 & 1 & 0 & 0 & 2 & 2 & 0 & lab \\
- & 21.1202 & 473.481 & 2 & $\mathrm{f}$ & 0 & 1 & 1 & 0 & 0 & 2 & 2 & 0 & lab \\
2 & 21.5625 & 463.769 & 1 & $\mathrm{f}$ & 0 & 1 & 1 & 0 & 0 & 2 & 0 & 0 & lab \\
1 & 21.6102 & 462.744 & 1 & $\mathrm{e}$ & 0 & 0 & 0 & 0 & 0 & 1 & 1 & 0 & lab \\
\hline
\end{tabular}

been found that $\mathrm{H}_{3}^{+}$can be the dominant positive ion in cool stellar gases in stars of less than $1 \times 10^{-3}$ times the solar metal abundance [13]. However, $\mathrm{H}_{3}^{+}$ is at best a trace species, with abundances typically around a few parts per billion, $\mathrm{H}_{3}^{+}$lines are therefore weak. Neale et al [53] calculated an ab initio line list of 3 million $\mathrm{H}_{3}^{+}$transitions. This line list has been extensively tested against a variety of laboratory data $[45,46,54]$ and found to perform well.

Figure 3 shows the synthetic stellar spectrum, computed using the Neale et al $\mathrm{H}_{3}^{+}$line list, of a star of surface gravity $\log g=1.0$, effective surface temperature $\mathrm{T}_{\text {eff }}=3750 \mathrm{~K}$ and metallicity, $Z$, of $3 \times 10^{-4}$ that of the Sun $\left(Z_{\odot}\right)$. The two spectra have been normalised by using a Planck function at $3750 \mathrm{~K}$ to provide a pseudo-continuum. Our calculations indicate that $\mathrm{H}_{3}^{+}$ should be detectable in stars of $\mathrm{T}_{\text {eff }}<4000 \mathrm{~K}$ and $Z / Z_{\odot}>5 \times 10^{-4}$. However, the number of stars known at present with such low metallicities is small; they are invariably dim and generally warmer than $4500 \mathrm{~K}$. These factors mean that it is very difficult to obtain high resolution, high signal to noise spectra of a sufficiently low metallicity star. The detection of $\mathrm{H}_{3}^{+}$in a stellar atmosphere may need to wait until a suitable candidate is discovered and identified.

\subsection{Water}

Water is probably the single most important molecule when considering the opacity of cool stars. It is not surprising, therefore, that there have been several attempts, known as MT [41], AMES [56], VTP1 [57] and SCAN [58] for example, to compute comprehensive line lists for use in models of stellar atmospheres. However none of these line lists have proved entirely satisfactory [59] 
1

2

3

4

5

6

7

8

9

10

11

12

13

14

15

16

17

18

19

20

21

22

23

24

25

26

27

28

29

30

31

32

33

34

35

36

37

38

39

40

41

42

43

44

45

46

47

48

49

50

51

52

53

54

55

56

57

58

59

60

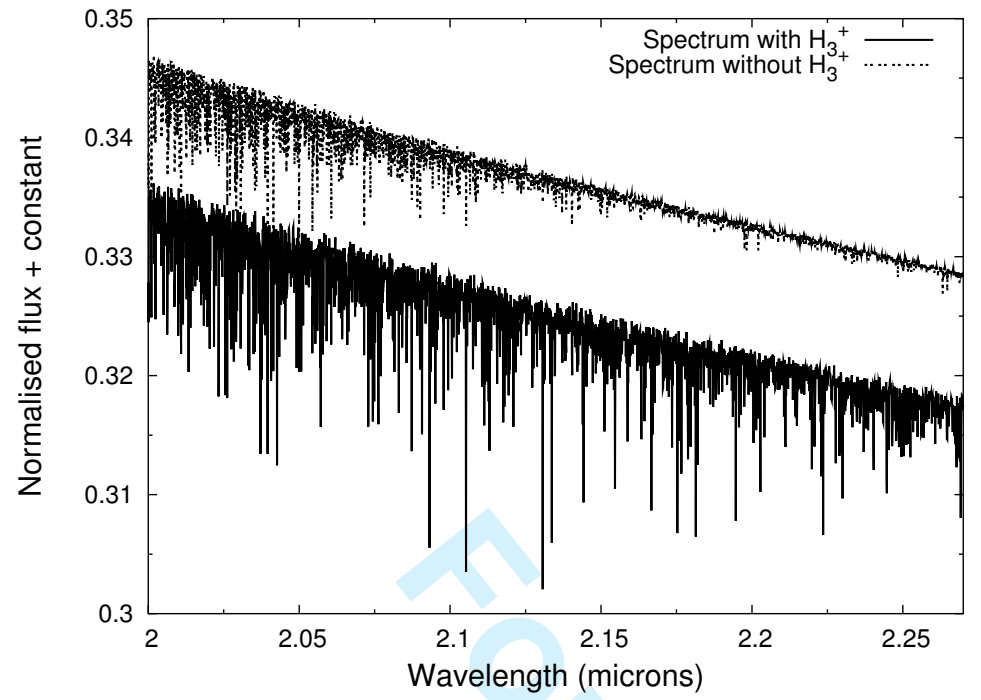

Figure 3. Synthetic spectra of a star of $\log g=1.0, \mathrm{~T}_{\text {eff }}=3750 \mathrm{~K}$ and metallicity of $3 \times 10^{-4}$ that of the Sun computed with and without $\mathrm{H}_{3}^{+}$lines. A constant of 0.01 has been added to the flux of the spectrum computed without $\mathrm{H}_{3}^{+}$lines to displace it from the other spectrum.

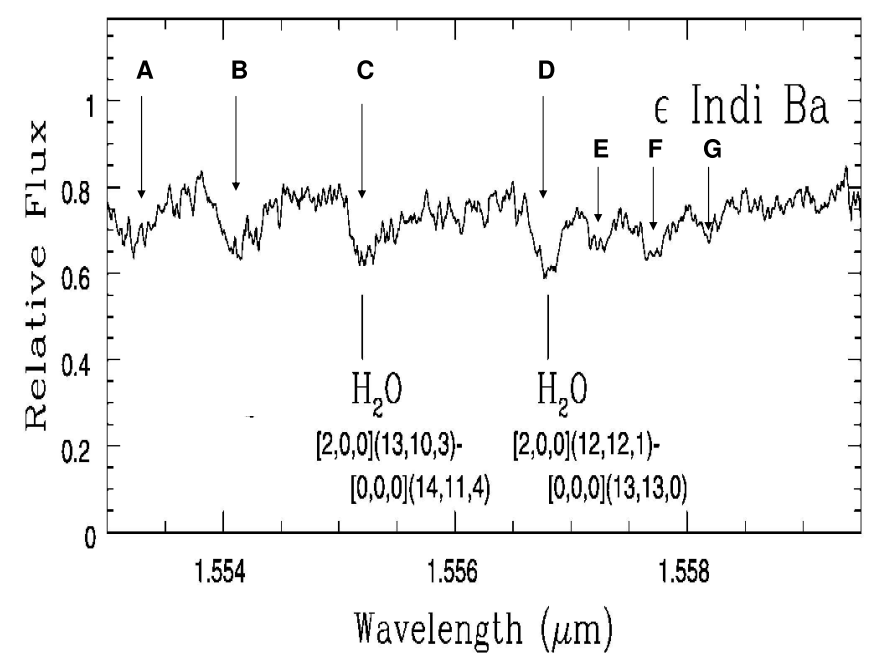

Figure 4. Spectrum of brown dwarf $\epsilon$ Indi Ba as reported by Smith et al [55]. Features labelled $\mathrm{H}_{2} \mathrm{O}$ were assigned in the orignal work; the letters refer to assignments presented in table 3. 
Table 3. Identification of the main lines in the seven principal absorption features in the spectrum of $\epsilon$ Indi $\mathrm{Ba}$ [55]. $\mathrm{x}$ indicates that the approximate quantum numbers are unknown. The wavelengths given here are in the rest frame. The observed data are blue shifted by approximately $80 \mathrm{~km} \mathrm{~s}^{-1}$. The letters given under observed features refer to Figure 4 .

\begin{tabular}{|c|c|c|c|c|}
\hline $\begin{array}{c}\text { Upper level } \\
\left(\nu_{1} \nu_{2} \nu_{2}\right)\left[\mathrm{JK}_{a} \mathrm{~K}_{c}\right]\end{array}$ & $\begin{array}{c}\text { Lower level } \\
\left(\nu_{1} \nu_{2} \nu_{2}\right)\left[\mathrm{JK}_{a} \mathrm{~K}_{c}\right]\end{array}$ & $\begin{array}{c}\lambda \\
\mu \mathrm{m}\end{array}$ & $\begin{array}{r}\mathrm{I}_{l i n e} \times 10^{-24} \\
\mathrm{~cm} \mathrm{~mol} \\
-1\end{array}$ & $\begin{array}{c}\text { Feature } \\
\text { (observed) }\end{array}$ \\
\hline$\left(\begin{array}{lll}2 & 0 & 1\end{array}\right)\left[\begin{array}{lll}20 & 0 & 20\end{array}\right]$ & $\left(\begin{array}{lll}1 & 0 & 0\end{array}\right)\left[\begin{array}{lll}21 & 0 & 21\end{array}\right]$ & 1.55370 & 11.51 & A \\
\hline$\left(\begin{array}{lll}0 & 0 & 2\end{array}\right)\left[\begin{array}{lll}12 & 6 & 7\end{array}\right]$ & $\left(\begin{array}{lll}0 & 0 & 0\end{array}\right)\left[\begin{array}{lll}13 & 9 & 4\end{array}\right]$ & 1.55370 & 6.23 & A \\
\hline$\left(\begin{array}{lllll}3 & 0 & 1\end{array}\right)\left[\begin{array}{lll}6 & 2 & 4\end{array}\right]$ & $\left(\begin{array}{lllll}2 & 0 & 0\end{array}\right)\left[\begin{array}{lll}7 & 2 & 5\end{array}\right]$ & 1.55373 & 4.45 & A \\
\hline$\left(\begin{array}{lll}1 & 2 & 0\end{array}\right)\left[\begin{array}{lll}13 & 4 & 9\end{array}\right]$ & $\left(\begin{array}{lll}0 & 0 & 0\end{array}\right)\left[\begin{array}{lll}14 & 5 & 10\end{array}\right]$ & 1.55375 & 11.44 & $\mathrm{~A}$ \\
\hline$\left(\begin{array}{lll}2 & 0 & 1\end{array}\right)\left[\begin{array}{lll}18 & 3 & 16\end{array}\right]$ & $\left(\begin{array}{lll}1 & 0 & 0\end{array}\right)\left[\begin{array}{lll}19 & 2 & 17\end{array}\right]$ & 1.55375 & 9.00 & A \\
\hline${ }^{1}\left(\begin{array}{lll}0 & 2 & 2\end{array}\right)\left[\begin{array}{lll}19 & 1 & 18\end{array}\right]$ & $\left(\begin{array}{lll}1 & 0 & 0\end{array}\right)\left[\begin{array}{lll}20 & 2 & 19\end{array}\right]$ & 1.55455 & 9.34 & B \\
\hline$\left(\begin{array}{lll}0 & 2 & 1\end{array}\right)\left[\begin{array}{lll}20 & 1 & 19\end{array}\right]$ & $\left(\begin{array}{lll}0 & 0 & 0\end{array}\right)\left[\begin{array}{lll}21 & 1 & 20\end{array}\right]$ & 1.55455 & 10.66 & $\mathrm{~B}$ \\
\hline$\left(\begin{array}{lll}0 & 4 & 1\end{array}\right)\left[\begin{array}{lll}13 & 1 & 12\end{array}\right]$ & $\left(\begin{array}{lll}0 & 2 & 0\end{array}\right)\left[\begin{array}{lll}14 & 1 & 13\end{array}\right]$ & 1.55456 & 5.42 & $\mathrm{~B}$ \\
\hline$\left(\begin{array}{lll}3 & 0 & 0\end{array}\right)\left[\begin{array}{lll}14 & 3 & 12\end{array}\right]$ & $\left(\begin{array}{lll}0 & 0 & 1\end{array}\right)\left[\begin{array}{lll}15 & 3 & 13\end{array}\right]$ & 1.55457 & 13.51 & B \\
\hline${ }^{2}\left(\begin{array}{lll}2 & 1 & 0\end{array}\right)\left[\begin{array}{lll}12 & 8 & 5\end{array}\right]$ & $\left(\begin{array}{lll}0 & 1 & 0\end{array}\right)\left[\begin{array}{lll}13 & 9 & 4\end{array}\right]$ & 1.55461 & 9.14 & B \\
\hline$\left(\begin{array}{lll}2 & 0 & 0\end{array}\right)\left[\begin{array}{lll}13 & 10 & 4\end{array}\right]$ & $\left(\begin{array}{lll}0 & 0 & 0\end{array}\right)\left[\begin{array}{llll}14 & 11 & 3\end{array}\right]$ & 1.55570 & 12.69 & $\mathrm{C}$ \\
\hline$\left(\begin{array}{lll}2 & 0 & 0\end{array}\right)\left[\begin{array}{lll}13 & 10 & 3\end{array}\right]$ & $\left(\begin{array}{lll}0 & 0 & 0\end{array}\right)\left[\begin{array}{lll}14 & 11 & 4\end{array}\right]$ & 1.55570 & 38.07 & $\mathrm{C}$ \\
\hline$\left(\begin{array}{lll}2 & 0 & 0\end{array}\right)\left[\begin{array}{lll}12 & 12 & 0\end{array}\right]$ & $\left(\begin{array}{lll}0 & 0 & 0\end{array}\right)\left[\begin{array}{lll}13 & 13 & 1\end{array}\right]$ & 1.55723 & 11.85 & $\mathrm{D}$ \\
\hline$\left(\begin{array}{lll}2 & 0 & 0\end{array}\right)\left[\begin{array}{lll}12 & 12 & 1\end{array}\right]$ & $\left(\begin{array}{lll}0 & 0 & 0\end{array}\right)\left[\begin{array}{lll}13 & 13 & 0\end{array}\right]$ & 1.55723 & 35.55 & $\mathrm{D}$ \\
\hline$\left(\begin{array}{lll}0 & 4 & 1\end{array}\right)\left[\begin{array}{lll}12 & 0 & 12\end{array}\right]$ & $\left(\begin{array}{lll}0 & 2 & 0\end{array}\right)\left[\begin{array}{lll}13 & 0 & 13\end{array}\right]$ & 1.55771 & 26.83 & $\mathrm{E}$ \\
\hline$\left(\begin{array}{lll}1 & 2 & 0\end{array}\right)\left[\begin{array}{lll}15 & 8 & 7\end{array}\right]$ & $\left(\begin{array}{lll}0 & 0 & 0\end{array}\right)\left[\begin{array}{lll}16 & 9 & 8\end{array}\right]$ & 1.55809 & 17.33 & $\mathrm{~F}$ \\
\hline$\left(\begin{array}{lll}x & x & x\end{array}\right)\left[\begin{array}{lll}23 & x & x\end{array}\right]$ & $\left(\begin{array}{lll}0 & 0 & 0\end{array}\right)\left[\begin{array}{lll}24 & 8 & 17\end{array}\right]$ & 1.55813 & 5.68 & F \\
\hline$\left(\begin{array}{lll}1 & 2 & 0\end{array}\right)\left[\begin{array}{lll}13 & 9 & 4\end{array}\right]$ & $\left(\begin{array}{lll}0 & 0 & 0\end{array}\right)\left[\begin{array}{lll}14 & 10 & 5\end{array}\right]$ & 1.55864 & 12.33 & G \\
\hline$\left(\begin{array}{lll}1 & 2 & 0\end{array}\right)\left[\begin{array}{lll}13 & 9 & 5\end{array}\right]$ & $\left(\begin{array}{lll}0 & 0 & 0\end{array}\right)\left[\begin{array}{lll}14 & 10 & 4\end{array}\right]$ & 1.55864 & 4.11 & G \\
\hline
\end{tabular}

and we have therefore recently completed the calculation of a new line list known as BT2 [60].

The BT2 line list is a comprehensive compilation of $\mathrm{H}_{2}{ }^{16} \mathrm{O}$ vibration-rotation transition frequencies and intensities. The list was produced using the DVR3D program suite [61] for the rotation-vibration nuclear motions and the spectroscopically determined potential energy surface of Shirin et al [62]. BT2 is the most complete water line list in existence, comprising over 500 million transitions (65\% more than any other list) and it is also the most accurate (over $90 \%$ of all known experimental energy levels are within $0.3 \mathrm{~cm}^{-1}$ of the BT2 values). Its accuracy has been confirmed by extensive testing against astronomical and laboratory data.

The line list has been used to identify and/or model individual water lines in a variety of objects including comets [63-65], sunspots $[47,66]$, an oxyacetylene torch at $3000 \mathrm{~K}[47,66]$ and the nova-like object V838 Mon [67]. Comparison of the observed intensities with those generated by BT2 enables water abundances and temperatures to be derived for these objects. 
Figure 4 shows part of the infrared spectrum of the brown dwarf $\epsilon$ Indi Ba as recorded by Smith et al [55]. Smith et al's models suggest this star has a temperature of $1500 \pm 100 \mathrm{~K}$. As shown in the figure, Smith et al identified two of the main absorption features with the water lines but left the other features unassigned. Synthetic spectra generated using BT2 suggest that in fact most of the features shown in Fig. 4 can be directly associated with absorptions by hot water. Our assignments to these features are given table 3; some features are actually blends of several transitions. It should be noted that labelling highly excited levels of water with the appropriate (rigid-rotor/harmonic oscillator or rigid-rotor/local mode) approximate quantum number remains difficult and some of the upper levels probed in this spectrum remain to be assigned. This does not invalidate the spectral model since BT2 uses all rigorous quantum numbers and contains all the information required for a complete spectral simulation.

The major motivation in calculating the BT2 line list was to provide an opacity for models of the atmospheres of M dwarf stars. BT2 has been included in the NextGen grid of Hauschildt and co-workers [68] and used for models of $\mathrm{M}$ dwarf atmospheres. Preliminary results of this study suggest that use of BT2 gives models very similar to those generated using the AMES [56] line list, suggesting that the perceived problem with water opacities may actually be due to some other aspect of the model such as the chosen chemical abundances. Full results of this study will be published elsewhere.

\section{$3.4 \quad C_{3}$}

A line list for the $\mathrm{C}_{3}$ molecule is still under construction at UCL. The goal is to study the infrared absorption spectrum of the $\mathrm{C}_{3}$ molecule in its ground electronic state for rotation-vibration states up to $12500 \mathrm{~cm}^{-1}$ above the ground state. This work is particularly aimed at helping model atmospheres of cool carbon-rich stars.

Astronomically $C_{3}$ was observed for the first time in 1882 by Huggins in emission from comets and later in absorption through its fundamental antisymmetric stretch band in the atmosphere of the cool carbon star IRC+10216. IRC+10216 is a carbon-rich Mira variable with an unusually thick and dense envelope of dust and molecules. For this reason it gives a natural laboratory for understanding interstellar and stellar chemistry [79]. Bending excitation of $\mathrm{C}_{3}$ have also been observed in IRC+10216 and in the massive dense molecular cloud Sagittarius B2, which has the richest concentration of molecules in the Milky Way [79]; it is has also been observed in the atmosphere of other carbon stars [80]. The $\nu_{3}$ fundamental antisymmetric-stretch band has also been detected in the outer atmosphere of cool AGB (asymptotic giant branch) carbon stars [50]. 
Table 4. Differences, in $\mathrm{cm}^{-1}$, between the experimental [71-78] and calculated vibrational energy levels. Calculations performed by Mladenovic [69] and Ahmed et al [70] are compared with our DVR3D calculations obtained respectively using the MLAD and adjusted AHM PES's.

\begin{tabular}{|c|c|c|c|c|c|c|c|}
\hline \multirow[b]{2}{*}{$\nu_{1}$} & \multirow[b]{2}{*}{$\nu_{2}$} & \multirow[b]{2}{*}{$\nu_{3}$} & \multirow{2}{*}{$\begin{array}{c}\text { Observed } \\
\mathrm{G}(\nu)\end{array}$} & \multicolumn{4}{|c|}{ Obs-calc } \\
\hline & & & & 69 & MLAD & {$[70]$} & AHM \\
\hline 0 & 2 & 0 & 132.7993 & 0.5 & 5.7 & 2.5 & 2.5 \\
\hline 0 & 4 & 0 & 286.11 & -2.7 & -1.9 & -0.7 & -0.6 \\
\hline 0 & 6 & 0 & 461.09 & -11 & -20.1 & 0.7 & 0.7 \\
\hline 0 & 8 & 0 & 647.59 & -15.1 & -56.4 & -1.2 & -1.2 \\
\hline 0 & 10 & 0 & 848.4 & -19.1 & & -2.0 & -2.1 \\
\hline 1 & 0 & 0 & 1224.2 & -5.3 & 1.9 & 5.2 & 5.1 \\
\hline 1 & 2 & 0 & 1404.1 & -1.0 & 7.3 & 1.8 & -1.5 \\
\hline 1 & 4 & 0 & 1590.05 & 0.1 & 3.4 & 0.1 & 0.1 \\
\hline 0 & 18 & 0 & 1773.37 & & & -0.1 & -1.8 \\
\hline 1 & 8 & 0 & 1990.52 & -6.6 & -43.6 & -3.4 & -3.5 \\
\hline 0 & 0 & 1 & 2040.019 & 0.6 & 3.3 & 2.0 & 2.0 \\
\hline 0 & 2 & 1 & 2133.8876 & 4.7 & -3.0 & 0.1 & 0.1 \\
\hline 1 & 10 & 0 & 2210.5 & -15.3 & & -0.7 & -0.9 \\
\hline 2 & 0 & 0 & 2435.2 & -10.5 & 5.4 & 2.5 & 2.4 \\
\hline 1 & 12 & 0 & 2439.9 & & & 1.4 & 1.0 \\
\hline 0 & 24 & 0 & 2575.92 & & & -0.4 & 1.0 \\
\hline 2 & 2 & 0 & 2656.33 & -3.9 & -15.3 & 0.4 & 0.3 \\
\hline 2 & 4 & 0 & 2876.9 & -0.4 & 6.9 & 1.9 & 1.8 \\
\hline 2 & 6 & 0 & 3099.9 & -1.6 & -3.6 & 3.3 & 3.1 \\
\hline 1 & 0 & 1 & 3259.9 & & & 1.2 & 1.1 \\
\hline 3 & 0 & 0 & 3636.1 & & & 0.1 & -0.1 \\
\hline 3 & 2 & 0 & 3894.3 & & & -0.1 & -0.4 \\
\hline 0 & 0 & 2 & 4035.369 & & & 0.4 & 0.1 \\
\hline 0 & 2 & 2 & 4110.886 & & & 2.4 & 2.2 \\
\hline 3 & 4 & 0 & 4146.3 & & & 2.5 & 2.2 \\
\hline 0 & 4 & 2 & 4211.327 & & & 3.9 & 3.6 \\
\hline 0 & 6 & 2 & 4339.419 & & & 5.1 & 4.6 \\
\hline 3 & 6 & 0 & 4392.78 & & & 2.4 & 2.0 \\
\hline 2 & 0 & 1 & 4459.3 & & & -0.8 & -1.2 \\
\hline 3 & 8 & 0 & 4641.03 & & & 2.9 & 2.4 \\
\hline 0 & 10 & 2 & 4651.6 & & & 3.4 & 2.3 \\
\hline 0 & 12 & 2 & 4832.5 & & & 3.4 & 2.0 \\
\hline 4 & 0 & 0 & 4828.7 & & & -1.3 & -1.9 \\
\hline 0 & 14 & 2 & 5029 & & & 4.4 & -2.1 \\
\hline 0 & 16 & 2 & 5236.6 & & & 4.1 & -2.0 \\
\hline 1 & 0 & 2 & 5265.4 & & & -7.7 & -8.3 \\
\hline 1 & 2 & 2 & 5367.2 & & & -7.1 & -8.0 \\
\hline 1 & 4 & 2 & 5495.4 & & & -5.6 & -6.4 \\
\hline 1 & 6 & 2 & 5643.5 & & & -4.1 & -5.1 \\
\hline 1 & 8 & 2 & 5809.1 & & & -2.4 & -4.0 \\
\hline 5 & 0 & 0 & 6013.6 & & & -1.7 & -3.1 \\
\hline 6 & 0 & 0 & 7191.3 & & & -0.9 & -4.8 \\
\hline 7 & 0 & 0 & 8361.5 & & & 0.3 & -6.4 \\
\hline
\end{tabular}

$C_{3}$ is linear but shows a high degree of floppiness, its bending fundamental is only $63 \mathrm{~cm}^{-1}$. Experimental studies by Northrup, Sears and Rohlfing [71-75] show that $\mathrm{C}_{3}$ has a very flat bending potential but no barrier to linearity for either the vibrational ground state or for either of the stretching fundamentals. At equilibrium $\mathrm{C}_{3}$ has no permanent dipole moment. It shows a strong 
bend-stretch interaction which is unusual for a linear molecule. Consequently, the spectrum of $C_{3}$ is complicated with many overtones, missing lines due to nuclear spin statistics and many hot bands even at room temperature. .

Rotation-vibration calculations for $\mathrm{C}_{3}$ are being performed using the DVR3D program suite [61]. For such calculations the quality of the potential energy surface (PES) sets the accuracy of the subsequent ro-vibrational transition frequencies. For this reason, much experimental and theoretical effort has been focused on determining the potential-energy function. A number of full, three-dimensional ground state PESs are available for the carbon trimer. We have been testing these to assess their suitability for use in opacity calculations. The surfaces studied include those due to Jensen [81], Mladenovic [69] (MLAD) and Ahmed et al [70] (AHM). These latter two surfaces were both constructed by fitting $a b$ initio energies. Both used the functional form:

$$
V=\sum_{i, j, k} C_{i, j, k} S_{1}^{i} S_{2}^{j} \theta^{k}
$$

where $S_{1}$ and $S_{2}$ are the symmetric and antisymmetric stretch coordinates respectively, and $\theta$ is the bond-angle. The large basis set and the inclusion of connected triple substitution in the MLAD PES allowed the reproduction of energy levels up to $3000 \mathrm{~cm}^{-1}$ above the zero-point energy with a standard deviation of $7.3 \mathrm{~cm}^{-1}$ compared to experiment. AHM's pure ab initio PES is not as accurate as that of MLAD's one (average error of $61.6 \mathrm{~cm}^{-1}$ ). However adjustments by fitting a few potential coefficients using available spectroscopic data enabled AHM to obtain a PES which gives vibrational levels up to 8000 $\mathrm{cm}^{-1}$ with a Standard deviation of only $2.8 \mathrm{~cm}^{-1}$. An ab initio dipole surface is also available [81].

Vibrational energy levels calculation with $\mathrm{J}=0$ obtained using DVR3D and the MLAD and AHM adjusted PESs are given in table 4. The table compares our results with those given by the authors, in general there is good agreement between results obtained using the same potential. No results are given for the MLAD PES for band origins above $3100 \mathrm{~cm}^{-1}$ since these results are increasingly erratic which is not surprising given the limited scope of the of the original determination. Our calculations were performed up to energy of $12500 \mathrm{~cm}^{-1}$ where a conical intersection with an electronically exited state PES forbids further calculations with the present method. For this energy range the AHM potential is the most reliable and it is this we will use for the full calculation.

To reproduce accurate spectra of cool stars atmosphere with the temperature range of $2000-4000 \mathrm{~K}$ it will be necessary perform calculations with high rotational quantum number. Given the low bending frequency hot bands arising from a range of excited bending states will make an important contribution 
to the opacity.

\subsection{HDO and the deuterium test}

Deuterium was produced in small quantities in the big bang and has been burnt in the interiors of stars and brown dwarfs ever since. However theory suggests that the least massive brown dwarfs do not burn deuterium as their cores do not become hot enough. It would be highly desirable to develop something called the 'deuterium test' which gives a clear spectroscopic signature for the presence of deuterium in such objects. This test could be used to show whether a brown dwarf was either too cool or too young to have burnt its deuterium [82]. In principle in this fashion it should be possible to establish some firmer observational limits on the primordial abundance of deuterium.

It has been suggested [83] that the HDO molecule should be a good candidate for the deuterium test. We have therefore undertaken a series of calculations aimed at producing an HDO line list of similar quality to the BT2 list for $\mathrm{H}_{2}{ }^{16} \mathrm{O}$. This HDO line list is aimed at modelling hot spectra rather than stellar opacities since the very small quantities of deuterium present in most stars means that the opacity contribution of HDO will necessarily be small.

Calculations on hot $\mathrm{HD}^{16} \mathrm{O}$ are much less advanced than those on $\mathrm{H}_{2}{ }^{16} \mathrm{O}$. Partridge and Schwenke [56] calculated $\mathrm{HD}^{16} \mathrm{O}$ line positions, and associated line intensities, up to $15700 \mathrm{~cm}^{-1}$; these calculations were repeated with better convergence by Tashkun and Tyuterev [84], who extended the range up to $17800 \mathrm{~cm}^{-1}$. Both calculations are based on the PES optimised to the experimental data by Partridge and Schwenke [56] and an ab initio dipole moment surface (DMS) [85]. The calculations reproduce energy levels, and hence line positions, to an accuracy useful for applications in astrophysics and atmospheric sciences since the observed minus calculated (Obs - Calc) line positions are in general very small and never exceed $0.7 \mathrm{~cm}^{-1}$. However the range of frequencies studies is insufficient for both applications (eg. [86]). For this reason we decided to perform new calculation based on the same PES and DMS but using an enlarged basis set.

HDO calculations have been performed in Radau coordinates with the DVR3D program suite [61] and the Partridge-Schwenke spectroscopically determined potential energy surface [56]. Given its reduced symmetry and increased mass, which increases the density of states, it requires significantly more computational effort to converge all energy levels of HDO up to some given energy than is required for $\mathrm{H}_{2} \mathrm{O}$. For the energy range considered, $\mathrm{H}_{2} \mathrm{O}$ calculations can be performed on a desktop machine but for HDO it was necessary to use UCL's 224 processor SUN cluster 'Keter'. The spectra have so far been generated for transitions involving states $J \leq 15$, which is sufficient for atmospheric purposes and for analysing spectra from cometary tails. Calcula- 


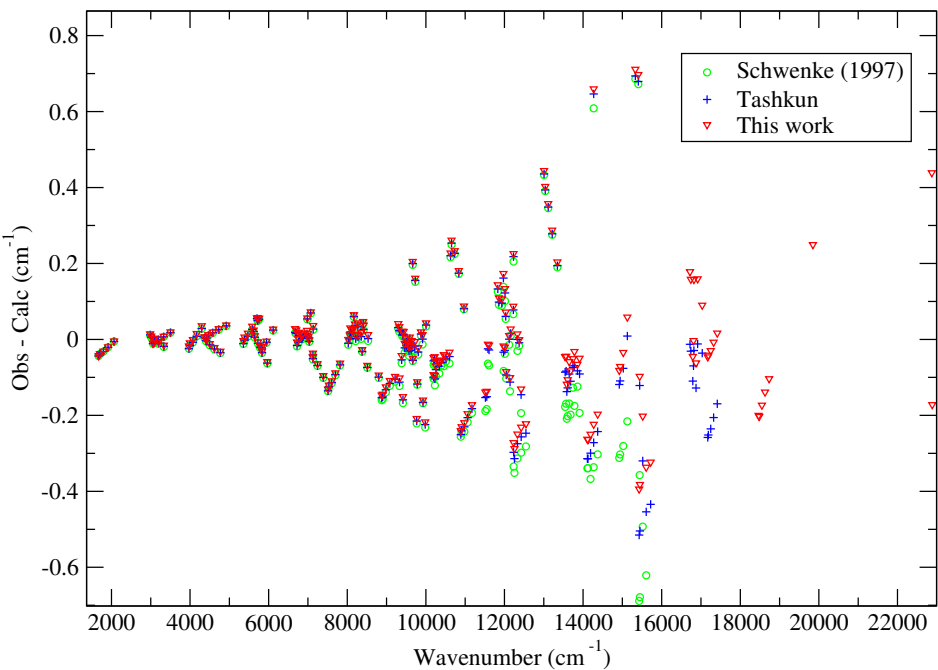

Figure 5. Comparison of calculated HDO energy levels with $J=5, p=1$ to experimental data [86-97] for this work and previous calculations $[56,84]$.

tions are continuing for higher $J$ states to allow higher temperature spectra to be synthesised.

A comparison between our calculations and previous studies for the observed vibrational band origins shows that all calculations reproduce the 88 measured band origins [86-97] to high accuracy but that our calculations marginally reduce the average error from $0.36 \mathrm{~cm}^{-1}$ for the previous two studies to 0.32 $\mathrm{cm}^{-1}$. Similar comparisons can be made for rotationally excited states.

Figure 5 compares our results with experiment and the previous studies $[56,84]$ over an extended energy range. It can be seen that up to 14000 $\mathrm{cm}^{-1}$ the calculations are in close agreement, above this level our new study gives levels which are systematically lower than the previous work. Given that the calculations are variational this is symptomatic of a lack of convergence in the previous studies. Above $20000 \mathrm{~cm}^{-1}$, for which there are only very limited experimental data, this difference becomes even more marked. For the range of energies considered all three calculations give similar agreement with experiment. As with other calculations discussed above, these differences are largely governed by the choice of PES. However for HDO there are also issues with failure of the Born-Oppenheimer approximation which need to be considered [98].

A problem with $\mathrm{HDO}$ as a marker for the deuterium test is the sheer number of lines in the spectrum of both $\mathrm{HDO}$ and $\mathrm{H}_{2} \mathrm{O}$ [99]. This means that despite the significant wavelength shift between the band centres in the two species, the HDO absorptions will still take place against a background of potentially much stronger $\mathrm{H}_{2} \mathrm{O}$ absorptions. For this reason other possible markers for the 
deuterium test are being sought.

Diatomic species have the advantage that they have fewer lines so the intensities should be concentrated in relatively few, stronger lines. Furthermore there are some metal hydrides that have bands in regions of suitable for ground based observations. A recent study [100] consider whether $\mathrm{CrH} / \mathrm{CrD}$ or $\mathrm{MgH} / \mathrm{MgD}$ might provide suitable species for a deuterium test. $\mathrm{CrH}$, for which both experimental $[101,102]$ and theoretical [103] laboratory studies have recently been reported, seems to be the more promising candidates. The detection of either $\mathrm{HDO}$ or $\mathrm{CrD}$ in the necessarily very faint spectrum of a brown dwarf remains extremely challenging and we will have to wait to see if either of these molecules provide a practical deuterium test.

\section{Conclusion}

As we have attempted to show in this paper significant progress has been made on determining line lists for use in constructing stellar opacities for key molecular species. However there remains much to be done before a level of completeness approaching that already achieved for hot stars [3-5] is obtained. In particular there are probably two areas in which significant extra work is required.

The first is rotation-vibration-electronic spectra of open shell diatomic systems. Although there are significant data on a few such systems, there are many potentially important ones, such as $\mathrm{FeO}$ [104] and $\mathrm{CaH}$ [105]. This is an area John Brown has contributed to significantly over many years.

The second area is that of the rotation-vibration spectra of key molecules with more than four atoms. We are just starting work on the ammonia molecule. Other important systems include acetylene and perhaps most vitally methane, for which some preliminary work is available [106]. In due course it might be necessary to consider other small systems such as formaldehyde [107].

Finally it should be mentioned that in stars cool there is the possibility of some of the molecules condensing onto grains [108]. Grains, whose spectra are generally rather poorly characterised, add yet another level of uncertainty to the already difficult task of modelling the atmospheres and spectra of cool stars.

\section{Acknowledgements}

We thank the many colleagues and collaborators that have contributed to our efforts to solve the opacity problem and in particular Roman Tolchenov for advice on labelling and Olga Naumenko for critically reading our manuscript. 
We would like to thank systems administrator Callum Wright for his help with many of the calculations discussed above. This work is supported by the UK Particle Physics and Astronomy Research Council, the Leverhulme Trust and the the European Union. BAV acknowledges financial support from the European Union for a Marie Curie Incoming Fellowships, grant WWLC008535.

\section{References}

[1] J. Tennyson. In Handbook of Molecular Physics and Quantum Chemistry, S. Wilson, ed., vol. 3, pp. 356-369. Wiley, Chichester (2003).

[2] Y. H. Le Teuff, T. J. Millar, A. J. Markwick. Astro. Astrophys., 146, 157 (2000).

[3] The Opacity Project Team. The Opacity Project: volume 1. Institute of Physics Publishing (1995).

[4] The Opacity Project Team, K. A. Berrington. The Opacity Project: volume 2. Institute of Physics Publishing (1997).

[5] F. J. Rogers, C. A. Iglesias. Astrophys. J. Suppl., 79, 507 (1992).

[6] R. L. Kurucz. Opacities for Stellar Atmospheres. Kurucz CD-ROM series. http://kurucz.harvard.edu (1991-1996).

[7] F. Allard, P. H. Hauschildt, D. R. Alexander, S. Starrfield. Ann. Rev. Astr. Astrophys., 35, 137 (1997).

[8] O. L. Polyansky, N. F. Zobov, S. Viti, J. Tennyson, P. F. Bernath, L. Wallace. Science, 277, 346 (1997).

[9] U. G. Jorgensen. In Molecules in Astrophysics: Probes and Processes, E. F. van Dishoeck, ed., vol. 178 of IAU proceedings, p. 441. Kluwer, Dordrecht (1997).

[10] R. Loidl, Lacon, U. G. Jorgensen. Astro. Astrophys., 371, 1065 (2001).

[11] H. R. A. Jones, Y. Pavlenko, S. Viti, R. J. Barber, L. Yakovina, D. Pinfold, J. Tennyson. Mon. Not. R. astr. Soc., pp. 105-112 (2005).

[12] K. Aoki et al. Astrophys. J., 639, 897 (2006).

[13] G. J. Harris, A. E. Lynas-Gray, S. Miller, J. Tennyson. Astrophys. J., 600, 1025 (2004).

[14] C. M. Sharp, A. Burrows. Astrophys. J. Suppl., in press (2007).

[15] P. Bergeron, M. T. Ruiz, S. K. Leggett. Astrophys. J. Suppl., 108, 339 (1997).

[16] G. J. Harris, A. Lynas-Gray, S. Miller, J. Tennyson. Astrophys. J., 617, L143 (2004).

[17] D. Saumon, P. Bergeron, J. I. Lunine, A. Burrows. Astrophys. J., 424, 333 (1994).

[18] S. Viti, H. R. A. Jones, P. Maxted, J. Tennyson. Mon. Not. R. astr. Soc., 329, 290 (2002).

[19] P. F. Weck, A. Schweitzer, K. Kirby, P. H. Hauschild, P. C. Stancil. Astrophys. J., 613, 567 (2004).

[20] A. E. Engel, N. Doss, G. J. Harris, J. Tennyson. Mon. Not. R. astr. Soc., 357, 471 (2005).

[21] U. G. Jorgensen. Astro. Astrophys., 284, 179 (1994).

[22] W. L. Virgo, T. C. Steimle, J. M. Brown. Astrophys. J., 628, 567 (2005).

[23] B. Plez. Astro. Astrophys., 337, 495 (1998).

[24] D. W. Schwenke. Faraday Discussions, 109, 321 (1998).

[25] Y. A. Pavlenko, H. R. A. Jones, Y. Lyubchik, J. Tennyson, D. Pinfold. Astron. Astrophys., 477,709 (2006).

[26] D. A. Fletcher, R. T. CARTER, J. M. Brown, T. C. STEImLE. J. Chem. Phys., 93, $9192(1990)$.

[27] J. P. Towle, J. M. BROWN, K. LIPUS, E. BACHEM, W. URBAN. Mol. Phys., 79, 835 (1993).

[28] R. T. CARTER, J. M. BROWN. J. Mol. Spectrosc., 166, 249 (1994).

[29] D. M. Goodridge, R. T. CARTER, J. M. BROWN, T. C. STEIMLE. J. Chem. Phys., 106, 4823 (1997).

[30] D. F. Hullah, C. Wilson, R. F. Barrow, J. M. BROWN. J. Mol. Spectrosc., 192, 191 (1998).

[31] D. M. Goodridge, D. F. Hullah, J. M. BROWN. J. Chem. Phys., 108, 428 (1998).

[32] D. F. Hullah, R. F. Barrow, J. M. BROWN. Mol. Phys., 97, 93 (1999).

[33] C. Wilson, J. M. BROWN. J. Mol. Spectrosc., 197, 188 (1999).

[34] C. Wilson, J. M. BROWN. J. Mol. Spectrosc., 209, 192 (2001).

[35] C. Wilson, J. M. BROWN. Mol. Phys., 99, 1549 (2001).

[36] C. Wilson, H. M. Cook, J. M. BROWN. J. Chem. Phys., 115, 5943 (2001).

[37] W. J. Balfour, J. M. BROWN, L. Wallace. J. Chem. Phys., 121, 7735 (2004). 
[38] J. M. BROWN, H. Korsgen, S. P. Beaton, K. M. Evenson. J. Chem. Phys., 124, 234309 (2006).

[39] T. C. STEIMLE, J. H. Chen, J. J. Harrison, J. M. BROWN. J. Chem. Phys., 124, 184307 (2006).

[40] M. Dulick, C. W. Bauschlicher Jr., A. Burrows, C. M. Sharp, R. S. Ram, P. Bernath. Astrophys. $J ., 594,651(2003)$.

[41] F. Allard, P. H. Hauschildt, S. Miller, J. Tennyson. Astrophys. J., 426, L39 (1994).

[42] G. J. Harris, Y. V. Pavlenko, H. R. A. Jones, J. Tennyson. Mon. Not. R. astr. Soc., 344, 1107 (2003).

[43] A. Burrows, D. Sudarsky, J. I. Lunine. Astrophys. J., 596, 587 (2003).

[44] L. Neale, J. Tennyson. Astrophys. J., 454, L169 (1995).

[45] H. Krechel, S. Krohn, L. Lammich, M. Lange, J. Levin, M. Scheffel, D. Schwalm, J. Tennyson, Z. Vager, R. Wester, A. Wolf, D. Zajfman. Phys. Rev. A, 66, 052509 (2002).

[46] H. Kreckel, D. Schwalm, J. Tennyson, A. Wolf, D. Zajfman. New J. Phys., 6, 151.1 (2004).

[47] P.-F. Coheur, P. F. Bernath, M. Carleer, R. Colin, O. L. Polyansky, N. F. Zobov, R. J. B. S. V. Shirin, J. Tennyson. J. Chem. Phys., 122, 074307 (2005).

[48] G. J. Harris, O. L. Polyansky, J. Tennyson. Astrophys. J., 578, 657 (2002).

[49] G. J. Harris, O. L. Polyansky, J. Tennyson. Spectrochimica Acta A, 58, 673 (2002).

[50] G. J. Harris, J. Tennyson, B. M. Kaminsky, Y. V. Pavlenko, , H. R. A. Jones. Mon. Not. R. astr. Soc., 367, 400 (2006).

[51] W. Aoki, T. Tsuji, K. Ohnaka. Astro. Astrophys., 340, 222 (1998).

[52] W. Aoki, T. Tsuji, K. Ohnaka. Astro. Astrophys., 350, 945 (1999).

[53] L. Neale, S. Miller, J. Tennyson. Astrophys. J., 464, 516 (1996).

[54] J. Tennyson, S. Miller. Spectrochimica Acta A, 57, 661 (2001).

[55] V. V. Smith, T. Tsuji, K. H. Hinkle, K. Cunha, R. D. Blum, J. A. Valenti, S. T. Ridgway, R. R. Joyce, P. Bernath. Astrophys. J., 599, L107 (2003).

[56] H. Partridge, D. W. Schwenke. J. Chem. Phys., 106, 4618 (1997).

[57] S. Viti, J. Tennyson, O. L. Polyansky. Mon. Not. R. astr. Soc., 287, 79 (1997).

[58] U. G. Jørgensen, P. Jensen, G. O. Sørensen, B. Aringer. Astro. Astrophys., 372, 249 (2001).

[59] H. R. A. Jones, Y. Pavlenko, S. Viti, J. Tennyson. Mon. Not. R. astr. Soc., 330, 675 (2002).

[60] R. J. Barber, J. Tennyson, G. J. Harris, R. N. Tolchenov. Mon. Not. R. Astr. Soc., 368, 1087 (2006).

[61] J. Tennyson, M. A. Kostin, P. Barletta, G. J. Harris, J. Ramanlal, O. L. Polyansky, N. F. Zobov. Computer Phys. Comm., 163, 85 (2004).

[62] S. V. Shirin, O. L. Polyansky, N. F. Zobov, P. Barletta, J. Tennyson. J. Chem. Phys., 118, $2124(2003)$.

[63] N. Dello Russo, M. A. DiSanti, K. Magee-Sauer, E. L. Gibb, M. J. Mumma, R. J. Barber, J. Tennyson. Icarus, 168, 186 (2004).

[64] N. Dello Russo, B. P. Bonev, M. A. DiSanti, E. L. Gibb, M. J. Mumma, K. Magee-Sauer, R. J. Barber, J. Tennyson. Astrophys. J., 621, 537 (2005).

[65] G. J. Harris, A. E. Lynas-Gray, S. Miller, J. Tennyson. Mon. Not. R. astr. Soc. 374, 337 (2007).

[66] N. F. Zobov, S. V. Shirin, O. L. Polyansky, R. J. Barber, J. Tennyson. J. Molec. Spectrosc., $237,115(2006)$.

[67] D. P. K. Banerjee, R. J. Barber, N. K. Ashok, J. Tennyson. Astrophys. J., 672, L141 (2005).

[68] P. H. Hauschildt, F. Allard, E. Baron. Astrophys. J., 512, 377 (1999).

[69] M. Mladenovic, S. Schmatz, P. Botschwina. J. Chem. Phys, 101, 5891 (1994).

[70] G. Ahmed, K. Balint-Kurti, C. Western. J. Chem. Phys, 121, 10041 (2004).

[71] F. J. Northrup, T. J. Sears. Chem. Phys. Lett., 159, 421 (1989).

[72] E. A. Rohlfing, J. E. M. Goldsmith. J. Chem. Phys., 90, 6804 (1989).

[73] F. J. Northrup, T. J. Sears. J. Opt. Soc. Am., 7, 1924 (1990).

[74] E. A. Rohlfing, J. E. M. Goldsmith. J. Opt. Soc. Am., 7, 1915 (1990).

[75] F. J. Northrup, T. J. Sears, E. A. Rohlfing. J. Mol. Spectrosc., 145, 74 (1991).

[76] K. Kawaguchi, K. Matsumura, H. Kanamori, E. Hirota. J. Chem. Phys., 91, 1953 (1989).

[77] C. A. Schmuttenmaer, R. C. COHEN, N. PUGLIANO, J. R. HEATH, A. L. COOKSY, K. L. BUSAROW, R. J. SAYKALLY. Science, 249, 897 (1990).

[78] J. Baker. J. Mol. Spectrosc., 183, 6 (1997).

[79] J. Cernicharo, J. R. Goicoechea. ApJ, 534, 199 (2000).

[80] R. Loidl, U. G. Hron, J.and Jorgense, S. Hofner. AA, 56, 1559 (1986).

[81] C. Jensen, P.and Rohlfing, J. Almof. J. Chem. Phys., 97, 3399 (1992).

[82] V. J. S. Bejar, M. R. Zapatero Osorio, R. Rebolo. Astrophys. J., 521, 671 (1999).

[83] G. Chabrier, I. Baraffe, F. Allard, P. H. Hauschild. Astrophys. J., 542, L119 (2000). 
[84] S. A. Tashkun, V. G. Tyuterev. http://spectra.ioa.ru.

[85] D. W. Schwenke, H. Partridge. J. Chem. Phys., 113, 16 (2000).

[86] B. A. Voronin, O. V. Naumenko, R. N. Tolchenov, J. Tennyson, S. Fally, P.-F. Coheur, M. Carleer, A. Jenovrier, A.C.Vandaele. J. Mol. Spectrosc., submitted (2006).

[87] R. A. Toth. J. Mol. Spectrosc., 195, 73 (1999).

[88] T. Parekunnel, F. Bernath, N. F. Zobov, S. V. Shirin, O. L. Polyansky, J. Tennyson. J. Molec. Spectrosc., 101, 28 (2001).

[89] A. Janca, K. Tereszchuk, P. F. Bernath, N. F. Zobov, S. V. Shirin, O. L. Polyansky, J. Tennyson. J. Molec. Spectrosc., 219, 132 (2003).

[90] O. V. Naumenko, I. Vasilenko, A.Campargue. J. Mol. Spectrosc., 234, 216 (2006).

[91] S. Hu, H. Lin, S. He, J. Cheng, Q. Zhu. Phys. Chem. Chem. Phys., 1, 3727 (1999).

[92] O. V. Naumenko, E. Bertseva, A. Campargue, D. W. Schwenke. J. Mol. Spectrosc., 201, 297 $(2000)$.

[93] O. V. Naumenko, E. Bertseva, A. Campargue. J. Mol. Spectrosc., 197, 122 (1999).

[94] O. V. Naumenko, A. Campargue. J. Mol. Spectrosc., 199, 59 (2000).

[95] O. N. Ulenikov, S.-M. Hu, E. S. Bekhtereva, Q.-S. Zhu. J. Mol. Spectrosc., 231, 57 (2005).

[96] O. V. Naumenko, O. Leshchishina, A. Campargue. J. Mol. Spectrosc., 236, 58 (2006).

[97] O. V. Naumenko, S.-M. Hu, S.-G. He, A. Campargue. Phys. Chem. Chem. Phys., 6, 910 (2004).

[98] N. F. Zobov, O. L. Polyansky, C. R. Le Sueur, J. Tennyson. Chem. Phys. Lett., 260, 381 (1996).

[99] Y. V. Pavlenko. Astron. Rep., 46, 567 (2002).

[100] Y. V. Pavlenko, G. J. Harris, J. Tennyson, H. R. A. Jones, J. M. Brown, J. Harrison, C. Hill. Mon. Not. R. astr. Soc. (2007).

[101] J. J. Harrison, J. M. Brown, D. T. Halfen, L. M. Ziurys. Astrophys. J., 637, 1143 (2006).

[102] P. K. Chowdhury, A. J. Merer, S. J. Rixon, P. F. Bernath, R. S. Ram. Phys. Chem. Chem. Phys., 8, 822 (2006).

[103] G. Ghigo, B. O. Roos, P. C. Stancil, P. F. Weck. J. Chem. Phys., 121, 8194 (2006).

[104] M. D. Allen, L. M. Ziurys, J. M. BROWN. Chem. Phys. Lett., 257, 130 (1996).

[105] J. H. Chen, J. Gengler, J. M. BROWN, T. C.Steimle. Phys. Rev. A, 73, 012502 (2006).

[106] A. Borysov, J. P. Champion, U. G. Jorgensen, C. Wenger. Mol. Phys., 100, 3585 (2002).

[107] R. Perez, J. M. Brown, Y. Utkin, J. X. Han, R. F. Curl. J. Mol. Spectrosc., 236, 151 (2006).

[108] D. R. Alexander, F. Allard, A. Tamanai, P. H. Hauschildt. Astrophys. Space Sci., 251, 171 (1997). 


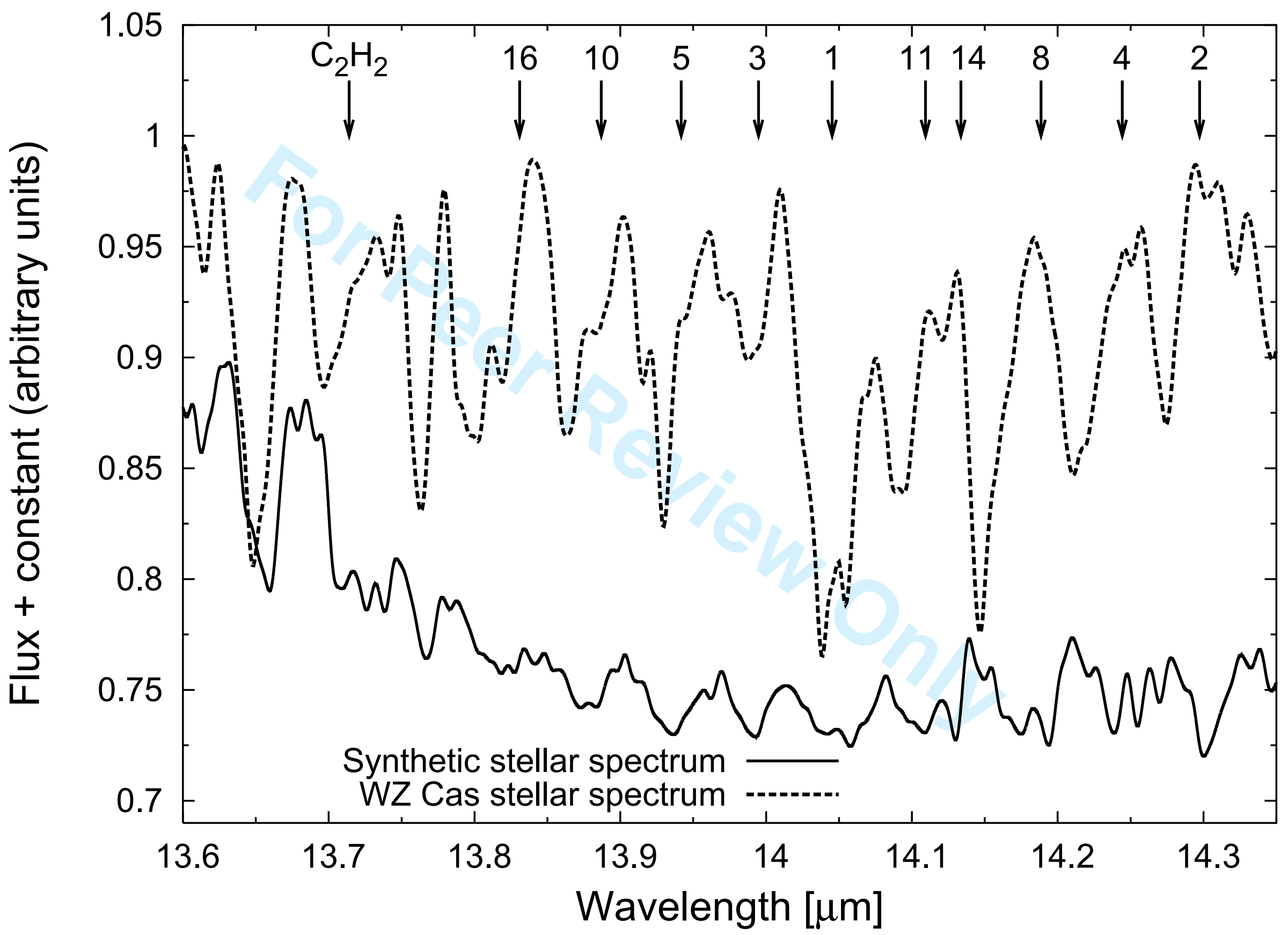

URL: http://mc.manuscriptcentral.com/tandf/tmph 


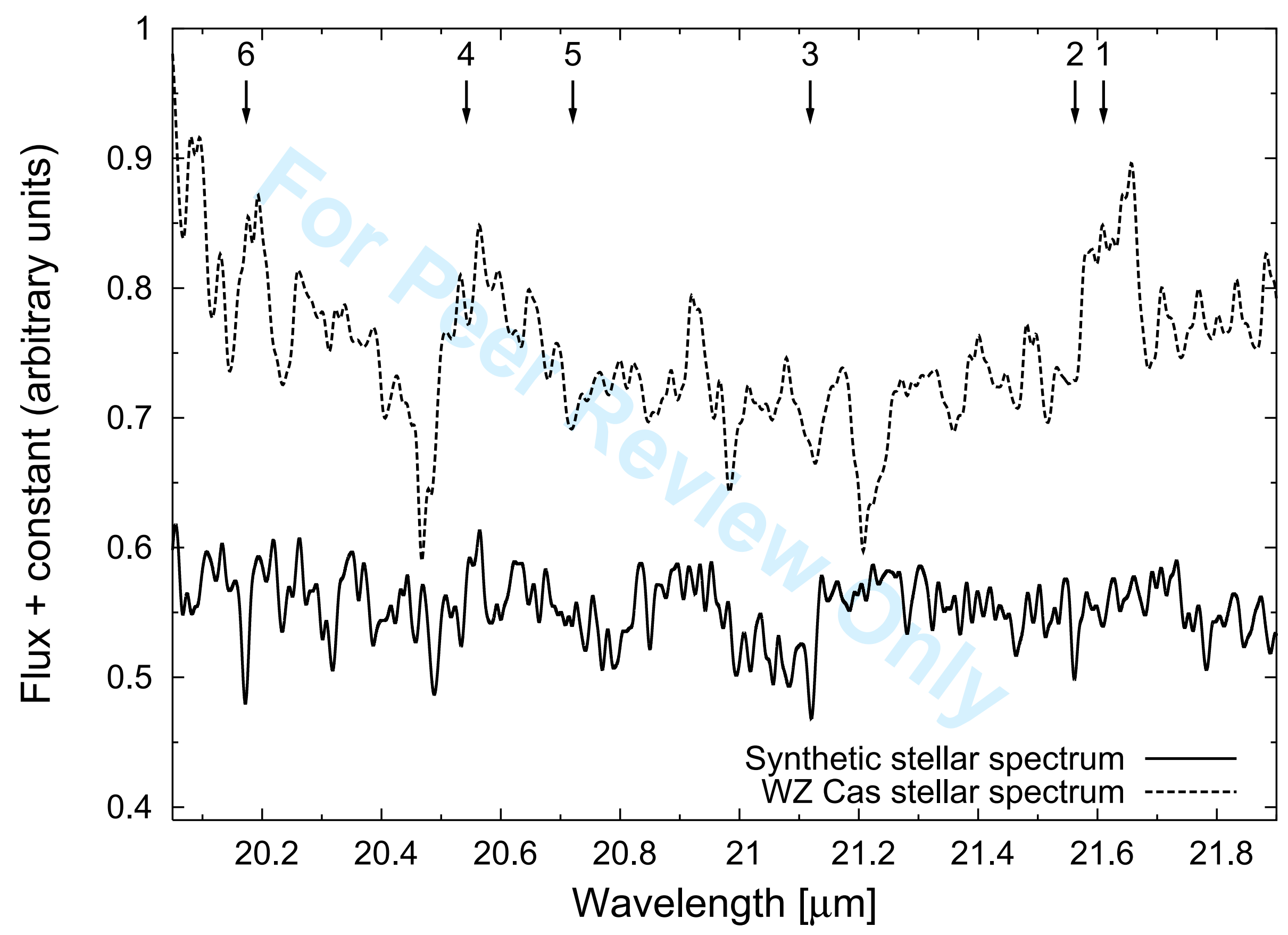

URL: http://mc.manuscriptcentral.com/tandf/tmph 


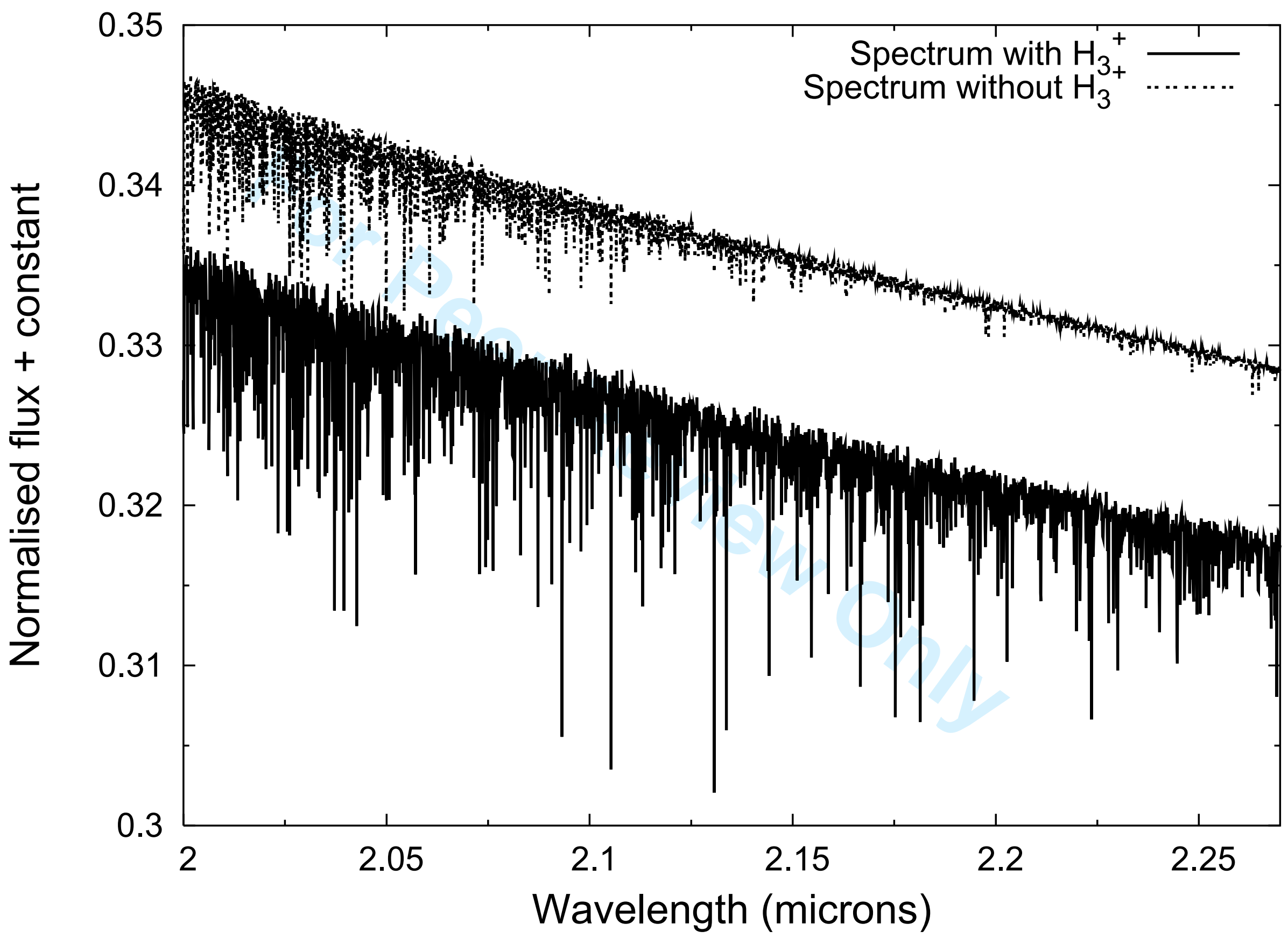




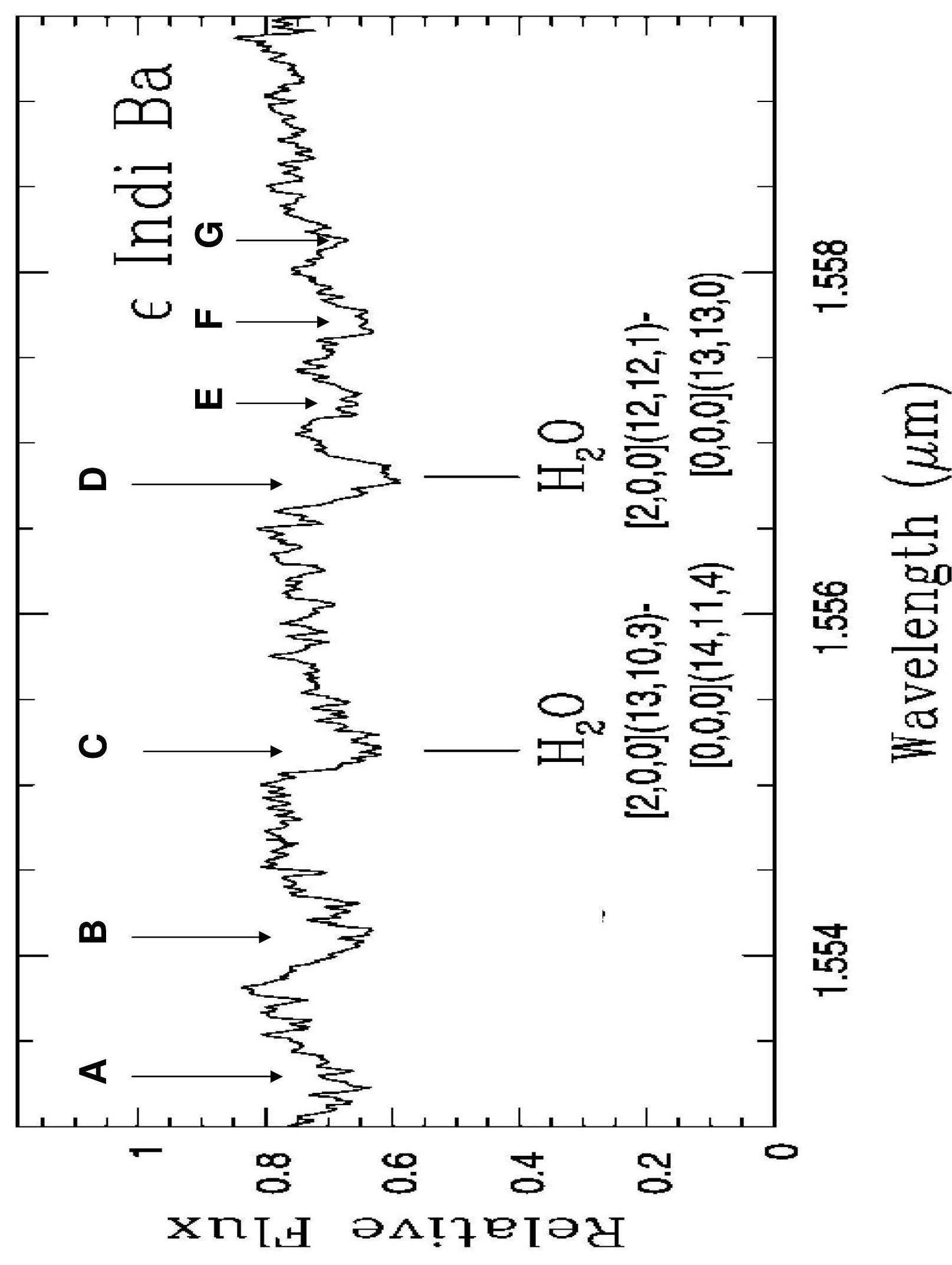

URL: http://mc.manuscriptcentral.com/tandf/tmph 


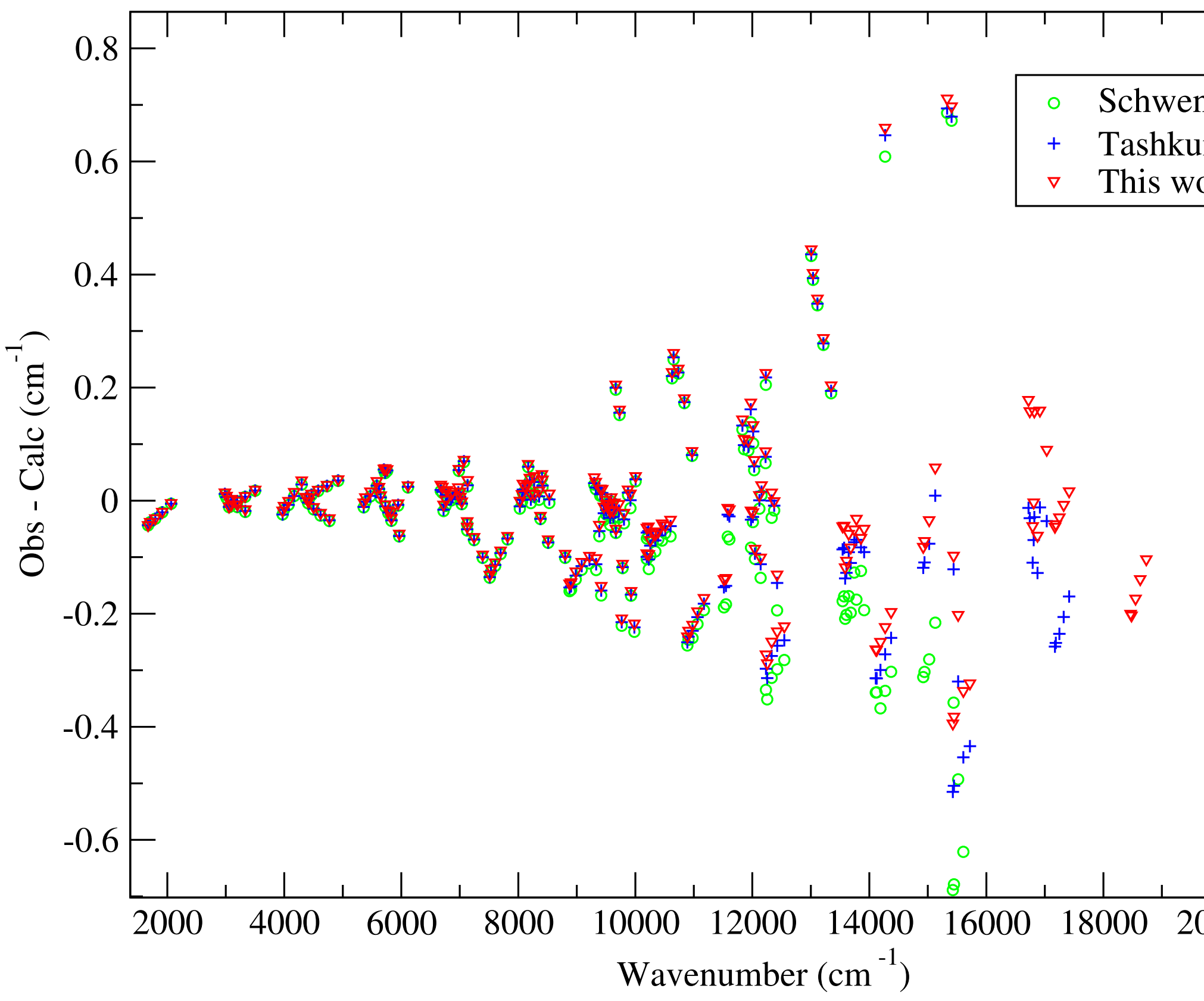

URL: http://mc.manuscriptcentral.com/tandf/tmph 